\title{
EL ANÁLISIS DE COMPONENTES PRINCIPALES EN LA INTERPRETACIÓN DE SISTEMAS AGROECOLÓGICOS PARA EL MANEJO DE RIZOBACTERIAS PROMOTORAS DEL CRECIMIENTO VEGETAL PARA EL CULTIVO DE LA CAÑA DE AZÚCAR
}

\author{
MAIN COMPONENTS ANALYSIS IN THE INTERPRETATION OF \\ AGROECOLOGICAL SYSTEMS FOR MANAGING RHIZOBACTERIA \\ IN THE GROWTH OF SUGARCANE CULTIVARS
}

MSc. Doris Torriente Díaz ${ }^{1}$; Dra Verena Torres Cárdenas ${ }^{2}$

\section{RESUMEN}

El Análisis de Componentes Principales constituye una herramienta estadística que tradicionalmente ha sido poco utilizada por los investigadores de la Rama Agrícola. En esta investigación se muestran las ventajas de su uso sobre otros métodos estadísticos y su utilidad en la interpretación de sistemas alternativos de nutrición de las plantas. Se experimentó sobre suelos Cambisoles en la cepa de retoños de caña de azúcar de más de tres cortes, evaluando el comportamiento de la planta ante diferentes formas de traslado al campo de las Rizobacterias Promotoras del Crecimiento Vegetal (líquido y sólidas). Las formas sólidas son soportadas en cachaza. Se obtuvo como resultados fundamentales que las variables longitud tallo, grosor del tallo, ancho de la hoja, no de hojas, área foliar entre otras, fueron las que más aportaron al sistema evaluado. Además se concluyó que las variables del tallo dentro de los sistemas biológicos como modo de nutrición de las plantas aportan mayor variabilidad a los indicadores productivos.

Palabras clave: Componentes Principales, Rizobacterias.

\section{ABSTRACT}

The Main Component Analysis constitutes a statistical tool that has traditionally been little used by agricultural researchers. This study shows the advantages of its use over other statistical methods and its usefulness in the interpretation of alternative nutrition systems in sugarcane. The experiment was carried out on Cambisol soils in the strain of regrowths of more than three cuttings, evaluating the performance of the plant before different transference forms of the Plant Growth Promoting Rhizobacteria to the field (liquid and solid). The solid forms are based on peat and sugarcane filtered mud. The main results obtained indicated that leaf length, stem width, leaf width, number of leaves, leaf area among others were the variables with the highest contribution to the evaluated system. It was also concluded that the biological systems for the nutrition of sugar cane contribute higher variability to the productive indicators.

Key words: Main components, Rhizobacteria.

\section{INTRODUCCIÓN}

El empleo de Rizobacterias Promotoras del Crecimiento Vegetal en el cultivo de la caña de azúcar soportado en líquido en determinadas con- diciones edafoclimáticas y de manejo agronómico ofrecieron resultados satisfactorios en el $76 \%$ de las experiencias realizadas, pero un $25 \%$ de los experimentos no se obtuvo la respuesta esperada en la planta, dado esto por múltiples razones. Dentro

\footnotetext{
Universidad de Matanzas. Autopista Varadero Matanzas Km 3 1/2. Matanzas. Cuba. doris.torriente@indio.atenas.inf.cu

2 Instituto de Ciencia Animal. vtorres@ica.co.cu
}

Fecha de Recepción: 26 Septiembre 2007

Fecha de Aceptación: 04 Enero 2008 
de ellas se destacan las relacionadas con los niveles de contaminación del producto y por problemáticas asociadas al manejo, almacenamiento y traslado de la bacteria al campo. (10)

Los estudios de las formulaciones recomendadas por los autores anteriores sugieren determinadas instrucciones necesarias a utilizar con estos productos como son: emplear para el almacenamiento y traslado al campo recipientes de acero inoxidable, de cristal o tanques plásticos limpios previamente tratados con vapor durante 15 minutos; debe existir la más exacta sincronización entre el fermentador y la aplicación al campo, para disponer de un producto de óptima calidad, ya que en estas condiciones se garantiza una estabilidad y pureza dentro de los 10 días después de recogida.

Ante estas limitaciones se llevó a cabo un estudio conjunto entre el Instituto de Derivados de la Caña de Azúcar (ICIDCA) y el Instituto de Investigaciones de la Caña de Azúcar (INICA) con el objetivo de formular un producto más estable que garantice una mayor conservación de la bacteria, un manejo más eficiente de la misma y que se adapte a las condiciones prácticas que exige el cultivo de la caña de azúcar.

Se llevaron a cabo estudios de optimización del medio y método de cultivo (3) de la bacteria sobre la formulación simplificada como una alternativa económica y disponible para uso industrial. Obtuvo una variante con la cepa Azospirillum brasilense 8I del INICA un producto con viabilidad $\left(10^{8} \mathrm{Ufc}\right.$ $\mathrm{m}^{-1}$ ) en un período de hasta 60 días.

Por su parte (12) patentizaron una tecnología de un producto a partir de la cepa (8I del INICA) soportada en sólidos (cachaza y turba) con grandes posibilidades de ser utilizada en caña de azúcar.

Para el estudio de estos soportes se utilizó como herramienta estadística el Análisis de Componentes Principales con el objetivo de establecer patrones de comportamiento en los sistemas ecológicos de nutrición de la caña de azúcar.

Partiendo de estos resultados y con la utilización de estos productos soportados en sólidos esta investigación se propone los siguientes objetivos:

1. Utilizar como herramienta estadística al ACP para establecer patrones de comportamiento de los sistemas ecológicos de nutrición de la caña de azúcar.

2. Evaluar de manera comparativa la respuesta fisiológica de la planta a las aplicaciones en soportes líquidos y sólidos (cachaza) de Rizobacterias.

\section{MATERIALES Y MÉTODOS}

\section{DESCRIPCIÓN DEL ÁREA DE ESTUDIO}

Los experimentos fueron montados en el Bloque experimental de la Empresa Cañera Sergio González de la provincia de Matanzas. Ubicado en los límites del Municipio de Perico, limita al norte con la Municipalidad de Martí, al oeste con el Poblado de Deseada, al este con la Empresa Cañera España Republicana y al sur con el Jagüey Grande.

Las variables climatológicas indican que los niveles de precipitaciones en esta área oscilan alrededor de los $1.500 \mathrm{~mm}$, la temperatura media promedio se enmarca entre $23,8{ }^{\circ} \mathrm{C}$ y la humedad relativa de $79 \%$ reportados en los boletines Agrometeorológicos de la Estación Indio Hatuey (1976 a 2005).

\section{MATERIAL UTILIZADO}

Se utilizaron vitroplantas de caña de azúcar en fase de adaptación de la variedad C. 120-78.

El producto utilizado fue obtenido de la cepa de Azospirillum brasilense 8I (INICA) en soporte líquido según metodología reportada por Roldós et al. (1994) y en soporte sólido según tecnología de Saura et al. (2003).

Para las aplicaciones de fertilizantes químicos se utilizaron los siguientes productos según dosis recomendadas por (11): Para nitrógeno la Urea, para fósforo el Superfosfato Triple y para potasio el Cloruro de potasio.

\section{DISEÑO EXPERIMENTAL}

Se diseñó según Bloque al Azar con cinco réplicas, donde los tratamientos consistieron en la combinación de los factores edad $(10,20,30$ y 40 días) y soportes de la Rizobacterias (80 1/ha de Azospirillum líquido, 80 1/ha de Azospirillum en cachaza, cachaza sola, fertilizantes químicos y control).

Se utilizaron 12 plantas por réplica, para un total de 60 para cada tratamiento, las vitroplantas se plantaron en potes de $40 \mathrm{~cm}^{3}$. Se aplicaron las siguientes cantidades del producto: para 80 1/ha líquido del preparado, 7,68 $\mathrm{ml}$ y para 80 1/ha del preparado sólido $2,4 \mathrm{~g}$. 


\section{METODOLOGÍA DE MUESTREO}

Se realizaron muestreos sistemáticos a los 50, 60, 70 y 80 días de edad de las plántulas a las siguientes variables respuestas:

- $\quad$ Longitud de la hoja +1 (según clasificación Kuiper citado por Dillewijn (1975)) en cm.

- Ancho de la hoja +1 en cm.

- Números de hojas activas, en unidades.

- Área foliar (longitud de la hoja*ancho de la hoja*número de hoja * 0,7) en $\mathrm{cm}^{2}$.

- Grosor del tallo en cm.

- Longitud del tallo en cm.

- Número de hojas.

- Crecimiento aparente (longitud inicial y longitud final desde la base del tallo hasta el último dewlap visible).

- Tiempo de emisión de las hojas (plastocrón) tiempo que transcurre durante la emisión de una hoja a otra) en días.

- Velocidad de Crecimiento (incremento en longitud por día) $\mathrm{cm} /$ día.

\section{ANÁLISIS ESTADÍSTICO}

Los resultados fueron sometidos a un análisis $\mathrm{ACP}$ al siguiente grupo de variables: longitud y ancho de la hoja, área foliar, grosor del tallo, número de hijos, longitud del tallo y tratamientos, para decidir cuáles son las variables de mayor importancia y las que aportaron a la variabilidad de los sistemas estudiados.

Se realizó además Análisis de Varianza según clasificación Doble con Interacción para los factores edad y soportes. En los casos de existir diferencia significativa se aplicó la Dócima de Comparación Múltiple de Duncan 1955.

\section{RESULTADOS Y DISCUSIÓN}

Para la selección de los valores propios significativos se partió del criterio Cliff (1987) citado (6), por el cual se adapta bien al objetivo del análisis. La descripción de los resultados se hará en función de los dos primeros componentes que explican el $78,66 \%$ de la varianza según tabla 19. Éstos dan una idea clara de la estructura que subyace en las variables estudiadas.
Tabla 1

Matriz de componentes y valores propios de las variables originales evaluadas en la aplicación de rizobacterias inoculadas en cachaza

\begin{tabular}{|l|c|c|}
\hline \multirow{2}{*}{ Variables } & \multicolumn{2}{c|}{ Componente } \\
\cline { 2 - 3 } & $\mathbf{1}$ & $\mathbf{2}$ \\
\hline Edad & $0,94_{1}$ & $-0,20$ \\
\hline Soportes & $-0,15$ & $-0,96_{1}$ \\
\hline Long. hoja & $0,77_{6}$ & $0,56_{2}$ \\
\hline Ancho hoja & $0,85_{4}$ & 0,35 \\
\hline No de hoja & $0,83_{5}$ & 0,43 \\
\hline Área foliar & $0,68_{7}$ & 0,35 \\
\hline Grosor tallo & $0,88_{3}$ & 0,35 \\
\hline No de hijo & 0,43 & 0,12 \\
\hline Long. tallo & $0,89_{2}$ & 0,32 \\
\hline Porcentaje de varianza & 67,05 & 11,61 \\
\hline Porcentaje de varianza acumulada & 67,05 & 78,66 \\
\hline
\end{tabular}

El primer componente principal contribuyó con más del $67 \%$ de la varianza total explicada, mientras que la distribución de los coeficientes del primer vector y de correlación indican, según tabla 21, que la edad de la planta, largo y grosor del tallo fueron las variables que más contribuyeron en forma positiva al componente, seguidas por ancho, número y largo de las hojas y por último el área foliar.

El segundo componente contribuyó solamente con el 11,6\% de la varianza total explicada y dentro de él la variable soportes fue la que más contribuyó de forma negativa; en segundo lugar, el largo de las hojas aportó sobre el resto de las variables una contribución destacada.

Esto nos indica que las variables asociadas al crecimiento del tallo mostraron los mejores resultados, dado esto por la etapa o fase de desarrollo objeto de estudio, es decir que la planta se encuentra en período de crecimiento, el cual se caracteriza por un encañamiento porque la planta comienza a formar los primeros canutos y comienzan a encañar y es donde la actividad fisiológica de la planta es máxima. Se destaca por parte de (5) que en esta fase ocurren fenómenos tales como la división, diferenciación y elongación celular. 


\section{LONGITUD TALLO}

La figura 1 muestra que para esta variable se encontraron diferencias significativas para la interacción variantes estudiadas y las edades de la planta analizadas. Se observa que a los 50 días se encontraron diferencias entre las variantes biológicas y las químicas y no así entre las aplicaciones sin y con cachaza, pero los mejores valores le correspondieron a las rizobacterias soportadas en sólidos.

A los 60 días de edad las plantas manifiestan diferencias altamente significativas con respecto a la edad anterior y entre las variantes biológicas y el testigo, los mejores valores le correspondieron a las aplicaciones de Azospirillum sin cachaza. Ya para los 70 días se observa el mayor valor en la inoculación soportada en sólido y al final de estudio, es decir a los 80 días de edad de las plántulas, se muestran diferencias altamente significativas entre la adicción de las rizobacterias sin y con cachaza, es decir, líquida y sólida. Esto es debido a las ventajas que ofrece el aplicar la bacteria soportada, pues el material orgánico le sirve de protección ante las dificultades que pueden encontrarse en el traslado al campo y en su manejo de forma general.

Se sugiere (3) que los formulados líquidos son las formas más simples de emplear los biopreparados. Es como tal que sale de los fermentadores, pero indica que es la menos apropiada, ya que requiere mover grandes volúmenes con peligro de contaminación en el transporte y almacenamiento y lo más importante es que el microorganismo llega al suelo desprovisto de protección y expuesto a los rigores del medio (calor, humedad, microflora, etc.), lo que disminuye la posibilidad de supervivencia.

Muchos autores recomiendan esta forma para casos donde no se pueda tratar la semilla botánica y que sea necesario aplicarlos al suelo directamente (4); refieren que la aplicación líquida de inoculantes micorrízicos es de hecho un reto debido a la baja protección osmótica de los propágulos aplicados en medio acuático y a sistemas de riego.

Nótese que entre las aplicaciones químicas y las de cachaza se han encontrado diferencias significativas y, cuando no ha sido así, los valores correspondientes al subproducto de la industria han

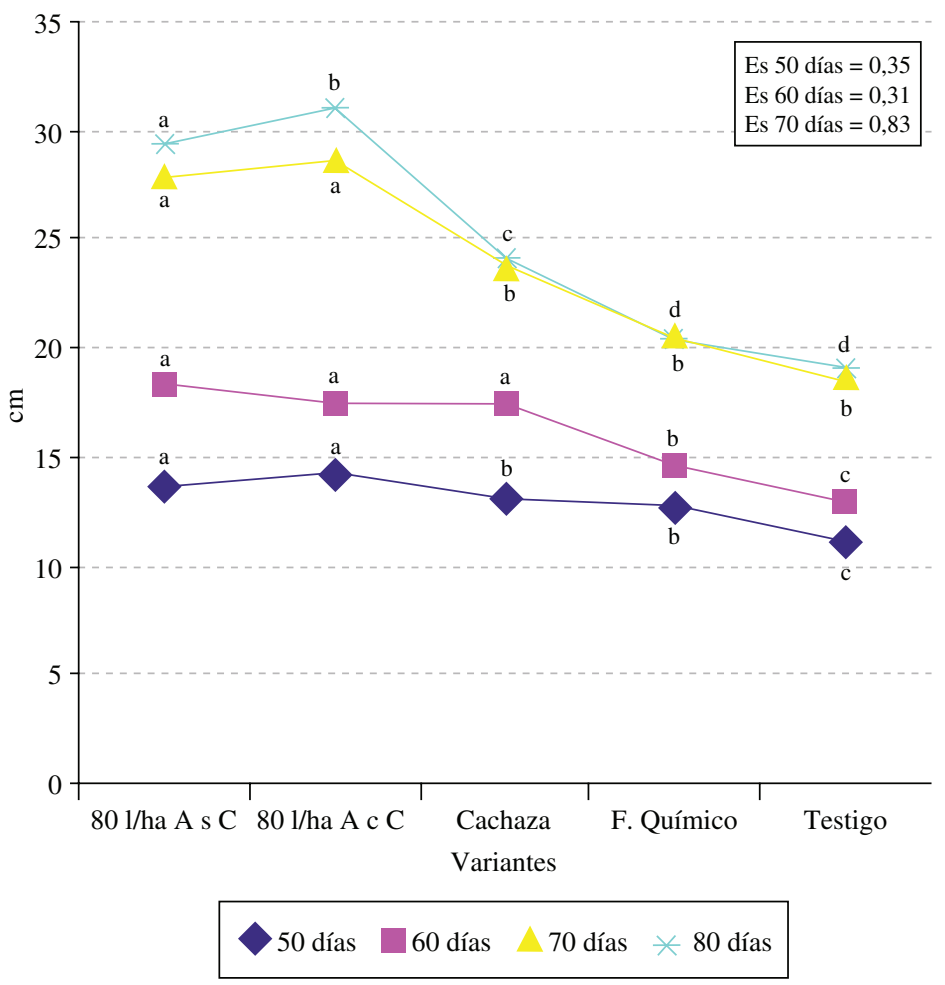

Figura 1. Comportamiento de la longitud del tallo para las variantes biológicas en las diferentes edades evaluadas. 
sido mayores. Este resultado muestra ventajas que posee la cachaza como suplemento de la nutrición química en muchos casos.

\section{GROSOR TALLO}

En la figura 2 se observa que para la variable grosor del tallo se encontraron diferencias altamente significativas entre las variantes y las edades estudiadas.

A los 50 días se hallaron diferencias entre las aplicaciones biológicas y los soportes utilizados (líquidos y sólidos), mostrando mejor valor las formas líquidas, mientras que para el resto de las edades no se muestran diferencias significativas entre el Azospirillum sin y con cachaza. Éstas a su vez sí se diferencian de las aplicaciones químicas y el testigo.

Estos resultados corroboran las ventajas que ofrece aplicar bacterias a los diferentes cultivos y las mismas pueden sustituir en muchos casos a las aplicaciones químicas con el consiguiente ahorro, por el gasto en que se incurre con los fertilizantes químicos y con el beneficio que las alternativas biológicas proporcionan al medio ambiente.

En el cultivo de la caña de azúcar se han estudiado ampliamente los beneficios que se obtienen con el empleo de la cachaza.

Algunos autores sugieren (7) que en muchos países su empleo es una práctica usual porque mejora las condiciones físicas y microbiológicas del suelo y suministra nutrientes. Destacan que $25 \mathrm{t} / \mathrm{ha}$ de cachaza equivalen a $154 \mathrm{~kg}$ de cloruro de potasio, $554 \mathrm{~kg}$ de superfosfato triple, $815 \mathrm{~kg}$ de urea y 15 t de materia orgánica.

\section{ANCHO DE LA HOJA}

Para esta variable, ver figura 3, a los 50 días no se encontraron diferencias significativas entre las variables estudiadas, ya para los 60 días se observan diferencias entre las variantes biológicas y el testigo. Para los 70 días entre las formas líquidas y sólidas si se hallaron diferencias, alcanzando los mayores valores la variante soportada en cachaza. Esta respuesta está avalada por el hecho reiterado anteriormente que las aplicaciones de Azospirillum promueven el crecimiento vegetal con resultados superiores en muchos casos a los obtenidos con la fertilización química y que las aplicaciones sólidas ofrecen mayor seguridad para el microorganismo.

$\mathrm{Al}$ respecto se estudió (2) en condiciones de laboratorio para la caña de azúcar a la cachaza como sustrato para el desarrollo de diferentes microorganismos como Azospirillum y se comprobó que la misma constituye un medio apropiado para el crecimiento y desarrollo de la bacteria.

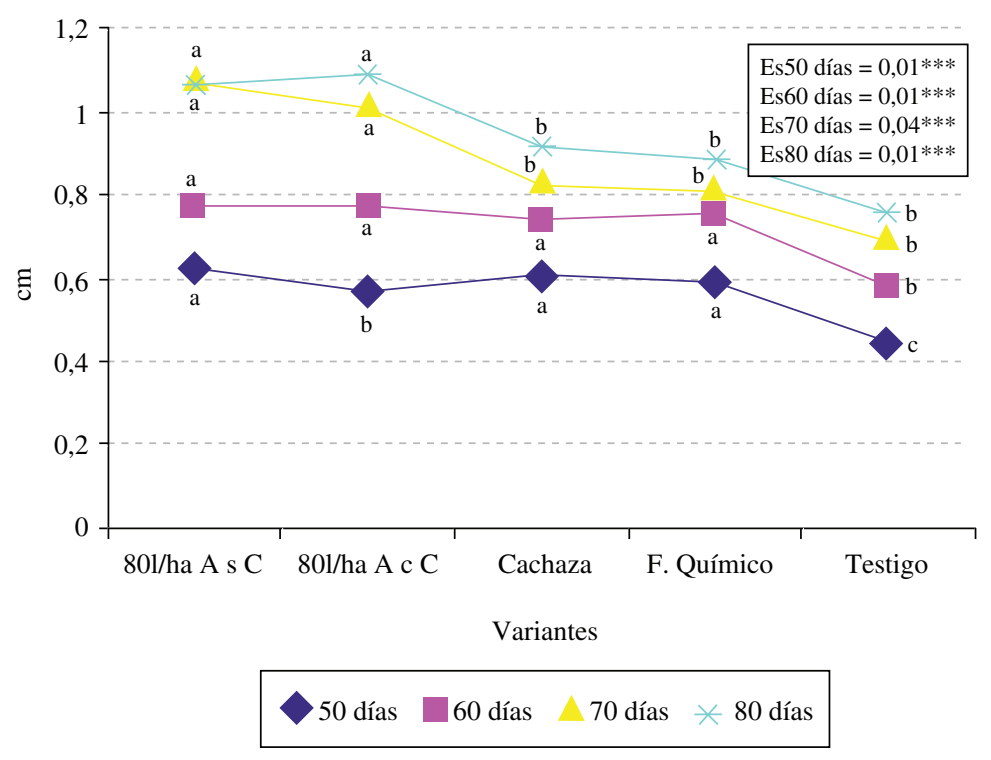

Figura 2. Comportamiento del grosor del tallo para las diferentes variantes y edades de las plántulas. 


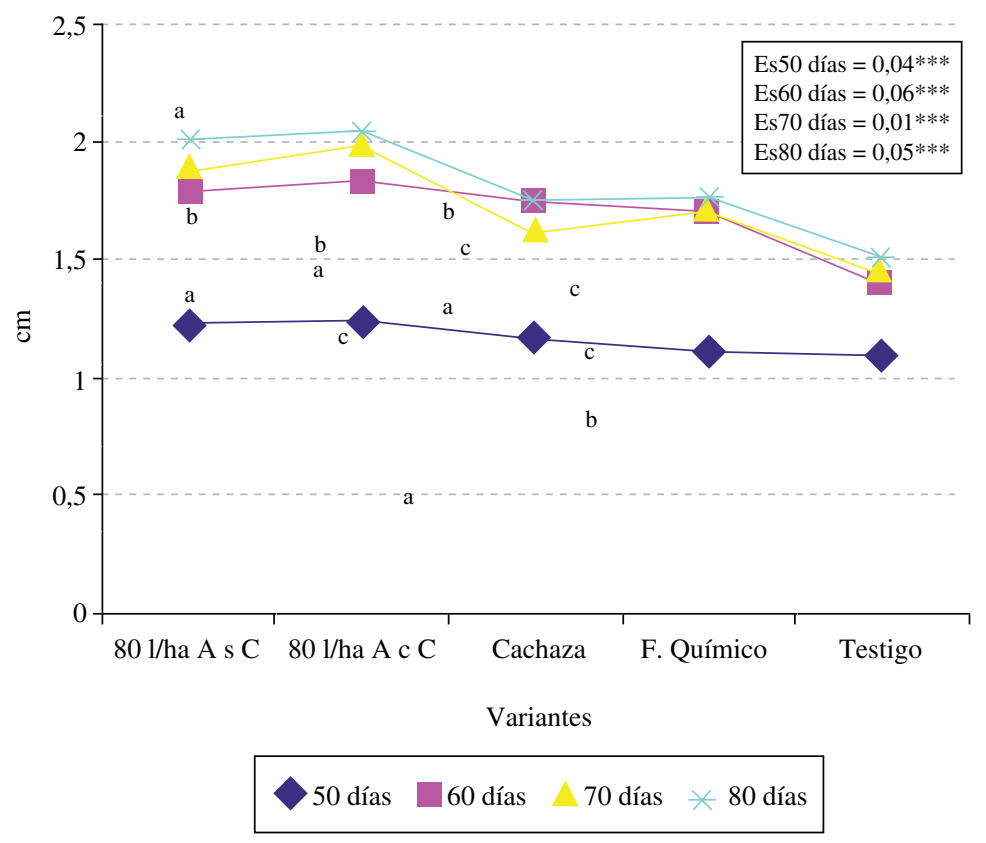

Figura 3. Comportamiento del ancho de las hojas para las diferentes formas de aplicación de Rizobacterias. Letras comunes no difieren. $\mathrm{P}<0.05$.

Destacó además (2) que este subproducto de la industria azucarera posee características como su facilidad de obtención, su bajo peso, posee determinados nutrientes que le permiten al microorganismo mantenerse vivo por más tiempo que en forma líquida, puede aplicarse de forma manual y mecanizada y su aplicación favorece en general a gran variedad de tipos de suelos.

\section{NÚMERO DE HOJAS}

La figura 4 muestra que esta variable a los 50 días mostró diferencias altamente significativas entre las variantes biológicas (líquidas y sólidas), la química y el testigo. En este caso la variante líquida alcanzó mayor valor que la sólida; situación similar se encontró a los 60 días de edad de las plántulas.

A los 70 días sin diferencias significativas entre ellas las aplicaciones sólidas mostraron mejor resultados que las líquidas. Resultado similar se obtuvo a la edad de 80 días de analizada.

No obstante a través de las edades estudiadas se puede observar que la variante Azospirillum con cachaza mostró un mayor incremento en el número de hojas (2 hojas) que la bacteria soportada en líquido (1,75 hojas), lo que muestra un mayor ritmo en el crecimiento en las primeras.

\section{LONGITUD DE LAS HOJAS}

La longitud de las hojas a los 50 días (ver figura 5) no mostró diferencia significativa para las formas soportadas líquidas y sólidas para las edades estudiadas. Se puede destacar que para las aplicaciones químicas y con cachaza sola no se encontraron diferencias. Todas las variantes mostraron diferencias con el testigo o control.

Cuando analizamos los incrementos encontrados en $\mathrm{cm}$ para cada variante los valores más altos se hallaron en el Azospirillum soportado líquido con una diferencia con respecto al químico de $12,05 \mathrm{~cm}$ promedio y para el testigo de $15,51 \mathrm{~cm}$, diferencias éstas importantes para la producción fotosintética en esta etapa.

\section{ÁREA FOLIAR}

La figura 6 muestra que para esta variable a los 50 días no se encontraron diferencias altamente 


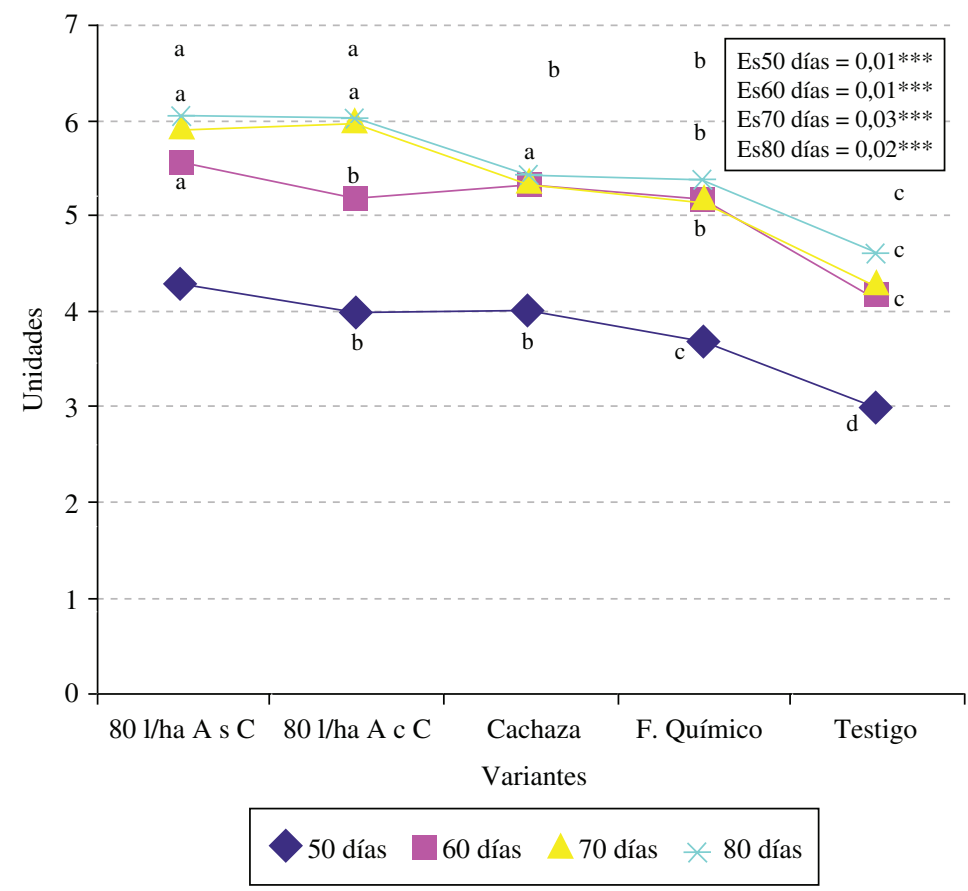

Figura 4. Comportamiento del número de hojas para las diferentes formas soportadas. Letras comunes no difieren. $\mathrm{P}<0.05$.

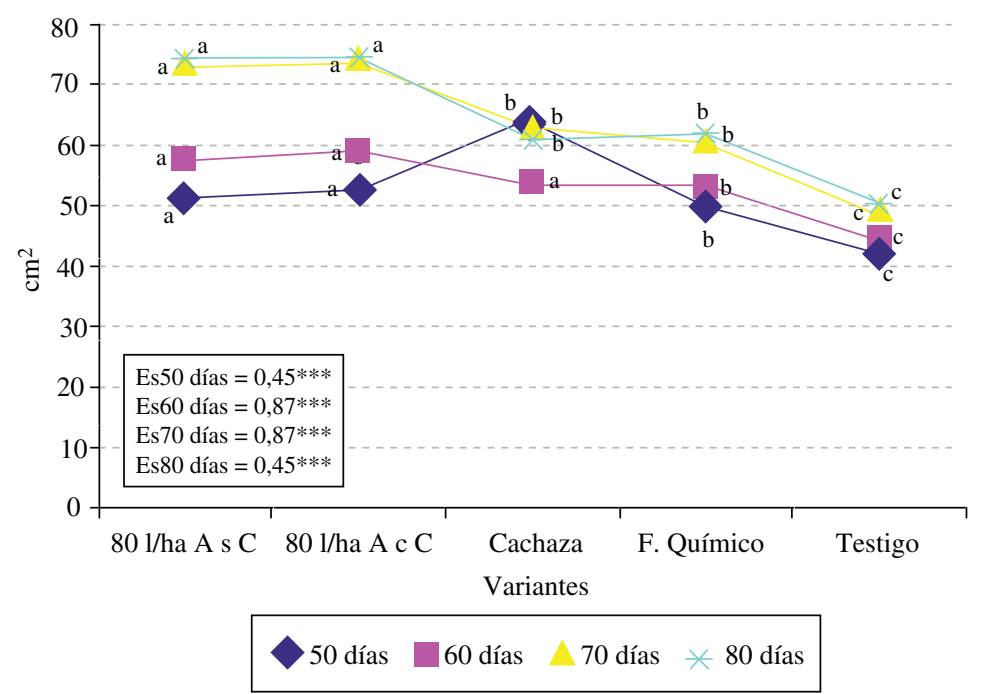

Figura 5. Comportamiento de la longitud de las hojas para las variantes biológicas. Letras comunes no difieren. Según dócimas Duncan para $\mathrm{P}<0.01$.

significativas entre las variantes estudiadas y con el testigo. A medida que se avanzó en edad esta condición sufrió variaciones y las alternativas biológicas se diferenciaron significativamente del resto de las variantes estudiadas.
A los 70 días se encontraron diferencias entre los soportes líquidos y sólidos alcanzando los mayores valores el Azospirillum con cachaza; ya a los 80 días las diferencias entre ellos no fueron significativas, aunque los incrementos en áreas para la variante 
soportada sólida alcanzó un valor $\left(443,20 \mathrm{~cm}^{2}\right)$ que duplica al área obtenida en el testigo o control y supera a la variante química.

Teniendo en cuenta que el análisis de componentes principales mostró que las variables relacionadas con el tallo juegan un papel muy importante en el sistema estudiado, la figura 7 muestra el crecimiento aparente y la velocidad del mismo para las variantes analizadas.

Se observa que el Azospirillum soportado en cachaza alcanzó los mayores valores. Esto se debe sin lugar a dudas a que la cachaza tiene un contenido de nutrientes que contribuye al desarrollo de la planta y además se puede aseverar que el microorganismo soportado en materiales orgánicos posee mejores condiciones de supervivencia que de forma líquida.

Estudios similares han demostrado las bondades que brinda la cachaza en este cultivo. (11); ha encontrado una estabilidad y viabilidad del Azospirillum en cachaza de $1,2 \times 10^{9}$ UfC hasta $2,7 \times 10^{8}$ durante 60 días, rango éste dentro de los parámetros establecidos para el uso de las bacterias como fertilizante biológico.

El ACP indicó que los estudios de la parte aérea de las plantas ocuparon el segundo lugar en importancia y dentro de ellos el área foliar fue uno de los que menos aportó a la variabilidad en el sistema, después de las variables relacionadas con el tallo. No obstante, las partes aéreas son las responsables de la producción de fotosintatos utilizados en el crecimiento y desarrollo de la planta.

En la figura 8 se muestra que los mayores incrementos en el área foliar alcanzados por las variantes Azospirillum sin y con cachaza, le correspondieron los menores tiempos de emisión de las hojas. El mayor tiempo de emisión de una hoja lo alcanzó el testigo al utilizar 13,8 días, con los menores incrementos de área en el follaje.

Se destaca (3) que este tiempo de emisión de las hojas (plastocrón) durante la fase inicial de la planta es muy breve -de dos a tres días- y en el gran período de crecimiento se puede extender de 6 a 15 días y que puede variar de acuerdo a las condiciones. Destaca a su vez que éste puede ser utilizado para caracterizar aspectos fisiológicos de la planta en determinada condición y ser relacionado con la productividad.

La variante con cachaza sola superó a la utilización de fertilizantes químicos. Esto demuestra que la cachaza posee propiedades químicas física que favorecen a las plantas, cuestión ésta reportada por diferentes autores como (1, 6 y 12).

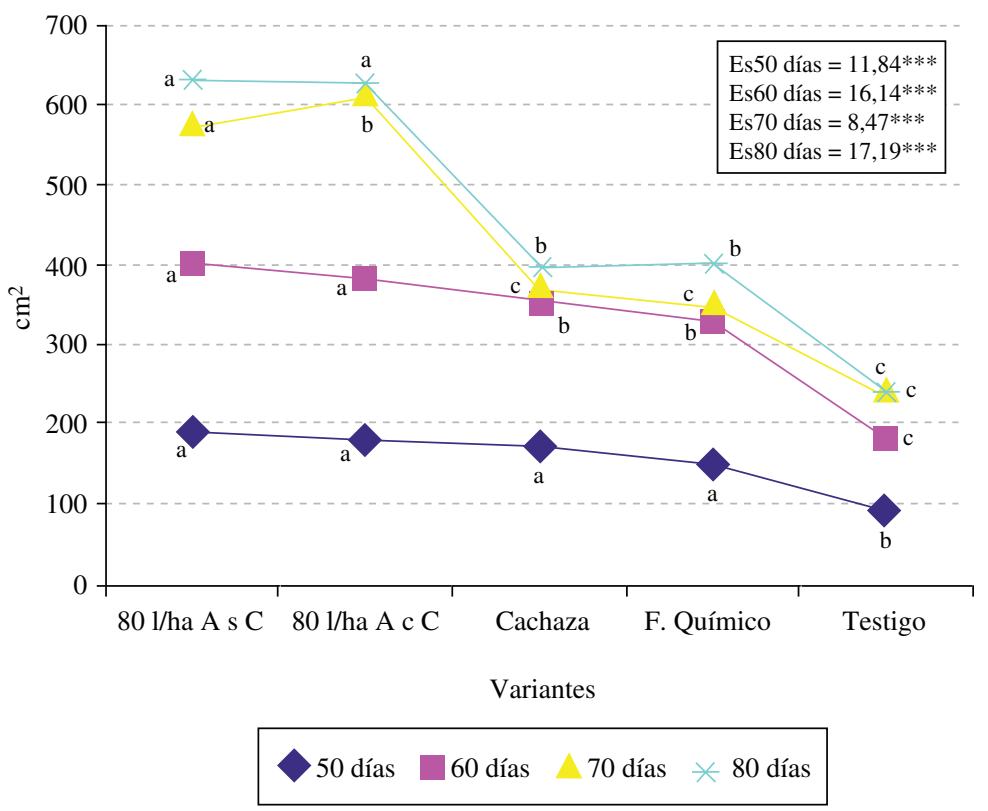

Figura 6. Comportamiento del área foliar para las variantes biológicas. Letras comunes no difieren según Duncan $\mathrm{P}<0,05$. 


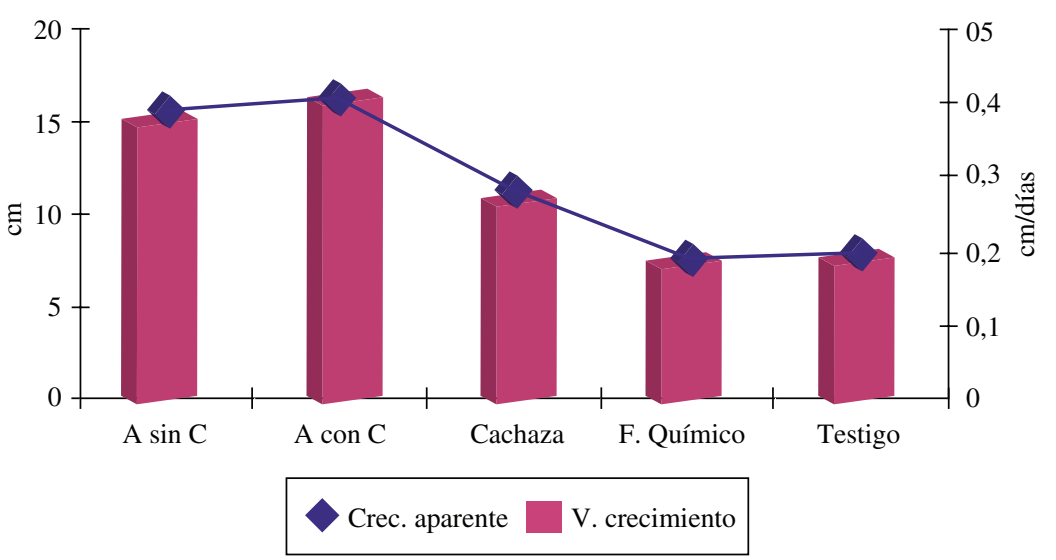

Figura 7. Crecimiento aparente y velocidad crecimiento del tallo para las variantes biológicas.

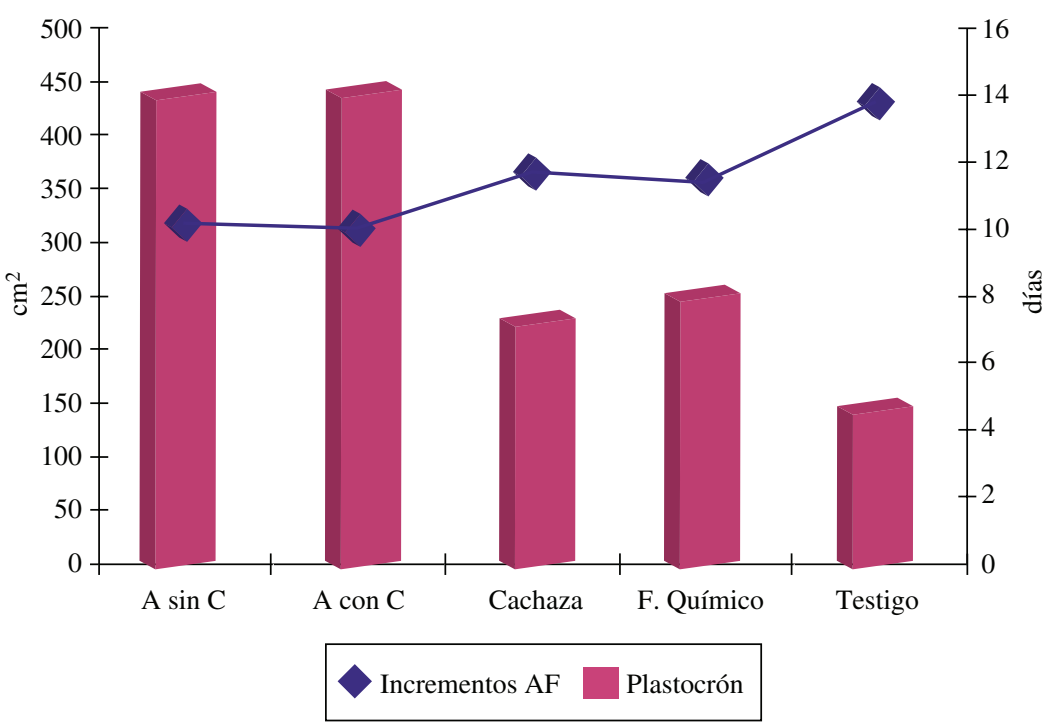

Figura 8. Relación del incremento del área foliar de las plantas con el tiempo de emisión de las hojas (plastocrón).

\section{CONCLUSIONES}

1. Que las variables longitud del tallo, grosor del tallo, ancho de la hoja, longitud de la hoja y área foliar, en ese orden, mostraron su influencia sobre la variabilidad del sistema.

\section{LITERATURA CITADA}

1. ARZOLA, N.; PANEQUE, H.; BATTLE, L.; MOREJÓN, C.; ALFONSO, B.; HERNÁNDEZ, G. 1990. La cachaza como enmienda orgánica y fertilizantes para la caña de azúcar. Folleto divulgativo. INCA. La Habana.
2. Que las variables relacionadas con el tallo aportaron mayor variabilidad explicada que las asociadas a las hojas.

3. Las variantes soportadas en cachaza mostraron mejores resultados que las soportadas en líquidos.

2. CARMENATE, $\mathbf{C}$. La producción de Azospirillum brasilence para el cultivo de la caña de azúcar. Tesis presentada en opción del título de ingeniero Agrónomo. Universidad de Matanzas. Camilo Cienfuegos. Pp. 46-58. 
3. FERNÁNDEZ, RAMONA. 1999. Obtención y Evaluación de un inoculante microbiano a partir de Azospirillum sp. Tesis (en opción al título de Máster en Ciencias Microbiológicas). Universidad de La Habana. Pp. 14-19, 20-26, 26-35, 36-59.

4. FERNÁNDEZ, L.; ORTEGA, E.; GRIMM, B.; HAINEZAL, M.R. 2006. Variación en la concentración de aminoácidos y azúcares en plantas de caña de azúcar inoculadas con Pantoea sp. Taller de biofertilización de los Trópicos". Congreso Científico del INCA (14: 2006, nov. 9-12, La Habana). Memorias. CD-ROM. Instituto Nacional de Ciencias Agrícolas. ISBN 959-7023-27-X

5. FERNÁNDEZ, R.; DÁVILA, A.; DEL TORO, F. 1983. Botánica y Fisiología de la Caña de Azúcar. Editorial. Pueblo y Revolución. Pp. 22, 120-153.

6. FRANCO, HIDALGO, R. (EDS.) 2003. Análisis Estadístico de Datos de Caracterización Morfológica de Recursos Filogenéticos. Boletín Técnico $\mathrm{N}^{\circ} 8$, Instituto Internacional de Recursos Filogenéticos (IPGRI), Cali, Colombia. 22, 27, 39.

7. GARCÍA DEL RISCO, E.; VÁZQUEZ FERNÁNDEZ, A. 2002. Los suelos y fertilización de la caña de azúcar. Instituto Nacional de Investigaciones de la Caña de Azúcar. Ciudad de La Habana. P. 46.

8. GUZMÁN,T.;SULROCA,F.; BOULART,L.;HERNÁNDEZ S. 1986. Agrotecnia de la Caña de azúcar III. Tomo I. Editorial ISAJAE. Ciudad de La Habana. P. 397-399.
9. HERNÁNDEZ, A; PLANA, R.; MARTÍN, G.; SANTANDER, J.L. 2002. Estudio de algunos géneros microbianos asociados a diferentes variedades de trigo (Triticum aestivum L.) en suelo Ferralítico Rojo. Rev. Cultivos Tropicales. Vol. 23, No 1, p. 15-20.

10. ROLDÓS, J.E.; CASA, M.; PÉREZ, J. 1994. Uso de los biofertilizantes en suelos Ferralíticos cultivados con caña de azúcar. Resúmenes. RECAR. La Habana. P. 9.

11. SERFE. 1996. Curso I. Los suelos dedicados al cultivo de la caña de azúcar. Instituto de Investigaciones de la Caña de Azúcar. P1, 31.

12. SAURA, G.; FERNÁNDEZ, R. 2003. Fijador de nitrógeno Azospirillum. En: www.fiago.org.sv. Rev. 5/11/2006.

13. TORRIENTE, DORIS. 2004. Aplicación de biofertilizante a base de Azospirillum soportado en turba en el cultivo de la caña de azúcar. En: Simposio internacional sobre caracterización y manejo de microorganismos rizosféricos y VII Taller de Biofertilización de los Trópicos. Congreso Científico del INCA (14:2004 nov. 9-12, La Habana). Memorias. CD-ROM. Instituto Nacional de Ciencias Agrícolas. ISBN 959-7023-27-X.

14. TRETO, EOLIA; GARCÍA, MARGARITA; BRUNET, R.; HERRERA, J.; KESSEL, J.; GÓMEZ, R.; IGLESIAS, R.; SANTANA, H. 1997. Nutrición y fertilización de la piña, 20 años de investigación en el Instituto Nacional de Ciencias Agrícolas. Cultivos Tropicales. 13(2-3) Pp. 5-59. 